\title{
THE HCN-WATER RATIO IN THE PLANET FORMATION REGION OF DISKS
}

\author{
Joan R. Najita ${ }^{1}$, John S. Carr ${ }^{2}$, Klaus M. Pontoppidan ${ }^{3}$, Colette Salyk ${ }^{1}$, \\ Ewine F. van DishoecK ${ }^{4}$, AND GeOfFrey A. BlaKe ${ }^{5}$ \\ ${ }^{1}$ National Optical Astronomy Observatory, 950 N. Cherry Avenue, Tucson, AZ 85719, USA \\ ${ }^{2}$ Naval Research Laboratory, Code 7211, Washington, DC 20375, USA \\ ${ }^{3}$ Space Telescope Science Institute, 3700 San Martin Drive, Baltimore, MD 21218, USA \\ ${ }^{4}$ Leiden Observatory, Leiden University, P.O. Box 9513, 2300 RA Leiden, Netherlands \\ ${ }^{5}$ Division of Geological \& Planetary Sciences, Mail Stop 150-21, California Institute of Technology, Pasadena, CA 91125, USA \\ Received 2012 September 26; accepted 2013 February 10; published 2013 March 15
}

\begin{abstract}
We find a trend between the mid-infrared $\mathrm{HCN} / \mathrm{H}_{2} \mathrm{O}$ flux ratio and submillimeter disk mass among T Tauri stars in Taurus. While it may seem puzzling that the molecular emission properties of the inner disk $(<$ few AU) are related to the properties of the outer disk (beyond $\sim 20 \mathrm{AU}$ ) probed by the submillimeter continuum, an interesting possible interpretation is that the trend is a result of planetesimal and protoplanet formation. Because objects this large are decoupled from the accretion flow, when they form, they can lock up water (and oxygen) beyond the snow line, thereby enhancing the $\mathrm{C} / \mathrm{O}$ ratio in the inner disk and altering the molecular abundances there. We discuss the assumptions that underlie this interpretation, a possible alternative explanation, and related open questions that motivate future work. Whatever its origin, understanding the meaning of the relation between the $\mathrm{HCN} / \mathrm{H}_{2} \mathrm{O}$ ratio and disk mass is of interest as trends like this among $\mathrm{T}$ Tauri disk properties are relatively rare.
\end{abstract}

Key words: circumstellar matter - protoplanetary disks - stars: pre-main sequence

Online-only material: color figures

\section{INTRODUCTION}

Emission from gaseous water and organic molecules such as $\mathrm{HCN}$ are common in the mid-infrared spectra of T Tauri stars, as measured with the Spitzer Space Telescope (Carr \& Najita 2011; Salyk et al. 2011; Pontoppidan et al. 2010; Pascucci et al. 2009). These emission features are believed to arise from the warm atmosphere of the disk within a few AU of the star, based on estimates of the temperature and emitting area of the emission (Carr \& Najita 2008, 2011; Salyk et al. 2008, 2011). This interpretation is supported by recent thermal-chemical models of gaseous inner disk atmospheres, which can account for the temperatures, column densities, and emitting areas of many of the observed molecular species (Najita et al. 2011; see also Agúndez et al. 2008; Woitke et al. 2009; Willacy \& Woods 2009; Heinzeller et al. 2011).

While molecular emission is common, the observed spectra of T Tauri stars show significant diversity. As one example, the water emission strength may be larger or smaller than that of HCN, depending on the source. Carr \& Najita (2011) noted that the flux ratio of $\mathrm{HCN}$ to $\mathrm{H}_{2} \mathrm{O}$ emission hinted at an increasing trend with disk mass, based on their small sample of objects. They noted that such a trend could result if higher mass disks are more efficient in forming large icy bodies ( $>\mathrm{km}$ in size; e.g., planetesimals and protoplanets) and consequently in locking up water in the outer disk. Because this sequestration of water in the outer disk (beyond the snow line) would reduce the water (and oxygen) abundance of the inner disk (e.g., Ciesla \& Cuzzi 2006), the inner disks of such systems would have an enhanced $\mathrm{C} / \mathrm{O}$ ratio and, consequently, higher abundances of organic molecules. Najita et al. (2011) showed that only modest enhancements in the $\mathrm{C} / \mathrm{O}$ ratio of the inner disk atmosphere are needed to produce a significant increase in the relative column densities of warm $\mathrm{HCN}$ and $\mathrm{H}_{2} \mathrm{O}$ in the disk atmosphere.
Finding possible evidence of planetesimal and protoplanet formation, even if indirect, is of interest as a test of planet formation theory, as planetesimals and protoplanets are the building blocks of ice and gas giant planets in core accretion theory. Currently, debris disks provide perhaps the best evidence for planetesimal formation outside the solar system (P. Armitage 2012 , private communication). The many debris disks that are now known suggest that planetesimals form efficiently and commonly, even though their formation mechanism remains uncertain (see Chiang \& Youdin 2010 for a review of our understanding of planetesimal formation). The high occurrence rate of extrasolar giant planets among mature solar-type stars further suggests that planetesimals and protoplanets must form commonly and within a Myr if core accretion is to operate within the few Myr gas dissipation timescale of disks (Hillenbrand 2008 and references therein).

One way to test this hypothesis is to look for evidence of planetesimal and protoplanet formation in Myr old protoplanetary disks. Here we take a step in this direction, by exploring in a larger sample the possible trend reported by Carr \& Najita (2011) involving the ratio of $\mathrm{HCN} / \mathrm{H}_{2} \mathrm{O}$ emission strength in T Tauri disks.

\section{OBSERVATIONS}

We obtained spectra of 18 classical $\mathrm{T}$ Tauri stars with Spitzer/IRS (Houck et al. 2004) as part of a GO-5 program (PID 50641; PI J. Carr). The results for one of the sources (DR Tau) has been discussed in previous reports on this program (Pontoppidan et al. 2010; Salyk et al. 2011). Because new observations in the IRS short-high module were not obtained for one of the GO-5 targets (IQ Tau), data for this star were obtained from the Spitzer archive (from PID 172; Evans et al. 2003; see Pontoppidan et al. 2010). These data were combined with data for 9 additional Taurus targets that were obtained in 
Table 1

Observations and Sample Properties

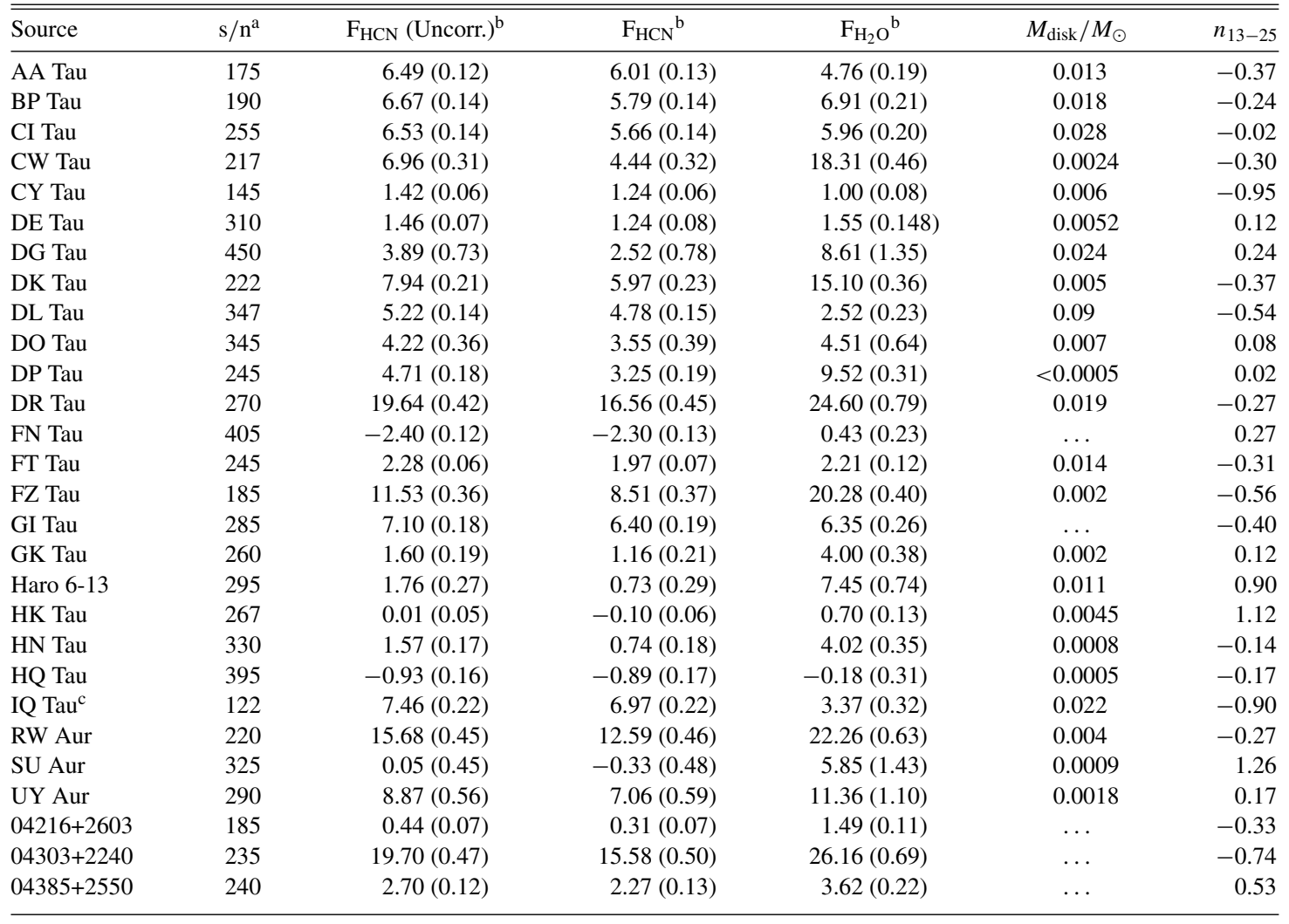

Notes.

a Average of the continuum signal-to-noise at $14 \mu \mathrm{m}$ and $17 \mu \mathrm{m}$.

${ }^{\mathrm{b}}$ Fluxes are in units of $10^{-17} \mathrm{~W} \mathrm{~m}^{-2}$. Errors are $1 \sigma$ uncertainties that only account for the noise in the spectrum.

c Archival data from the Spitzer c2d program (Evans et al. 2003).

a previously reported GO-2 program (PID 2300; Carr \& Najita $2011,2008)$ for a total sample of 28 objects.

All of the sources reported here are located in the TaurusAuriga star forming region. We focused on the Taurus sources from among the GO-5 targets, because they have submillimeter disk masses that have been estimated in a homogeneous way (Andrews \& Williams 2005). Because the targets are all wellknown members of Taurus, we can assume that the sample is a coeval population that originated from cloud cores of similar metallicity.

Our sample (Table 1) was chosen to span a broad range of stellar accretion rates, X-ray luminosities, and mid-infrared colors, but only a limited range in spectral type (primarily K3-M1). To characterize mid-infrared color, we used the spectral index between $13 \mu \mathrm{m}$ and $25 \mu \mathrm{m}, n_{13-25}$, as defined and reported by Furlan et al. (2006). The sample excludes T Tauri stars with the extreme mid-infrared colors of transition objects (roughly $n_{13-25}>1.5$; Furlan et al. 2006). Most sources are contained within the mid-infrared color bounds of "normal T Tauri stars" (roughly $n_{13-25}<0.5$ ) as denoted by Furlan et al. (2006), although a few have intermediate mid-infrared colors (Haro 6-13, HK Tau, and SU Aur; $n_{13-25}$ of $0.5-1.5$ ). The submillimeter disk masses of the sources also span a broad range. Table 1 gives the disk masses derived by Andrews \& Williams (2005), primarily from spectral energy distribution (SED) fits, assuming a gas-to-dust mass ratio of 100 .

None of the sources is known to have a companion close enough to dynamically alter the inner few AU of the disk, the region from which the Spitzer molecular emission arises (Carr \& Najita 2008, 2011; Salyk et al. 2011). However, several of the sources have companions close enough to be included in the 4".7-wide slit of IRS. Carr \& Najita (2011) discuss the companion properties of the GO-2 sources DK Tau, RW Aur, and UY Aur. The other targets with nearby companions (GK Tau at 2.5, HK Tau at 2".3, and HN Tau at 3". 1) have flux ratios that are large, so the companion is not expected to contribute significantly to the Spitzer spectrum. The companions to HK Tau and HN Tau are, respectively, $\sim 30$ times fainter at $11.8 \mu \mathrm{m}$ (McCabe et al. 2003) and $\sim 65$ times fainter at $L$ (White \& Ghez 2001). The faint optical companion to GK Tau (e.g., Hartigan et al. 1994), which may be physically unrelated (White \& Ghez 2001; Itoh et al. 2008), is $\sim 60$ times fainter than GK Tau at $H$ (Itoh et al. 2008) and is not detected with high-resolution MIR imaging (Skemer et al. 2011).

The spectra presented here were obtained with Spitzer IRS using the short-high $(\mathrm{SH} ; 10-19 \mu \mathrm{m})$ module, which has a nominal resolving power of $\sim 600$. The spectra were obtained in staring mode, in which the target is placed at two nod positions along the length of the $11^{\prime \prime}$ by $4^{\prime \prime} .7$ slit. High signalto-noise spectra were obtained through the use of a large number of nod cycles. The resulting redundancy allowed us to evaluate the noise statistics and identify badly behaved pixels. Details of the data reduction procedure are given in Carr $\&$ Najita (2011). Table 1 gives a representative value of the signal-to-noise of the resulting spectra. In obtaining dedicated background observations for all targets, we avoided positions 

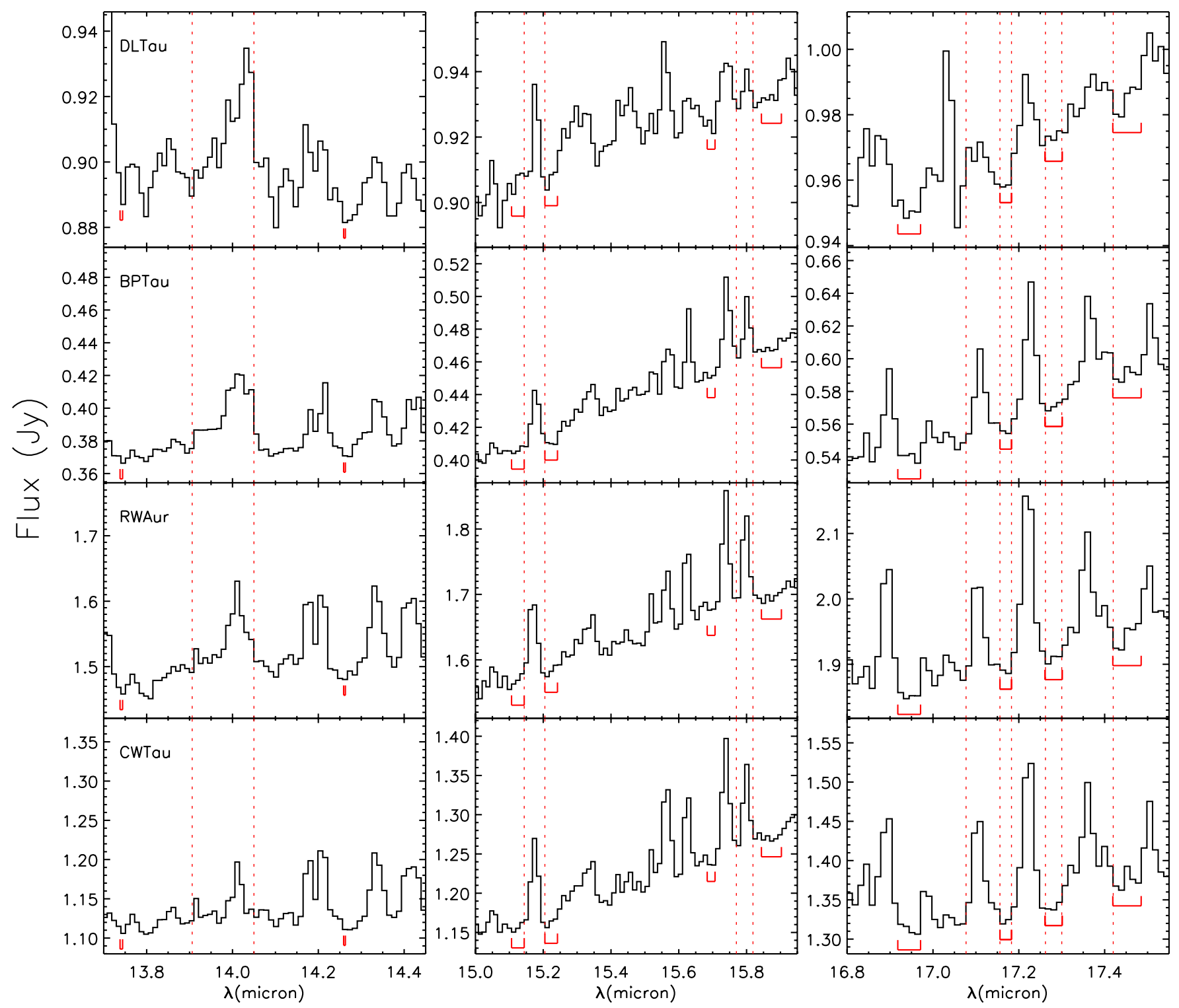

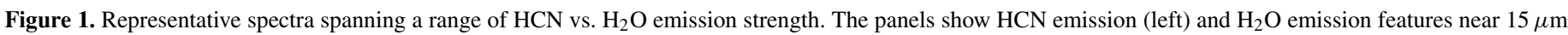

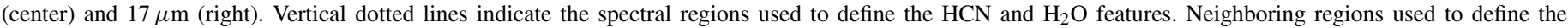
continuum are indicated by brackets below the spectrum.

(A color version of this figure is available in the online journal.)

with continuum sources and known nebulosity. In most cases, the background integration time was one-half of the time spent observing the target.

Here we report on the relation between $\mathrm{HCN}$ and $\mathrm{H}_{2} \mathrm{O}$ emission and disk mass. We will provide a more in-depth description of the results for all of the detected features in the SH and long-high ( $\mathrm{LH} ; 19-27 \mu \mathrm{m})$ modules in a subsequent report.

\section{RESULTS}

\section{1. $\mathrm{HCN}$ and $\mathrm{H}_{2} \mathrm{O}$ Emission Strengths}

As found in previous studies, molecular emission is common in our sample and the emission spectra are rich. In particular, emission features of $\mathrm{HCN}$ and $\mathrm{H}_{2} \mathrm{O}$ are commonly detected (Figure 1). Among the many water emission features present, the $17 \mu \mathrm{m}$ group of features $(17.12 \mu \mathrm{m}, 17.22 \mu \mathrm{m}, 17.36 \mu \mathrm{m})$ is an attractive measure of the water emission strength because these features are commonly detected, bright, and easy to measure, i.e., they are not blended with emission from other atoms or molecules. These features have been previously used to characterize the water emission from disks (Carr \& Najita 2011; Pontoppidan et al. 2010). Each feature is composed of multiple $\mathrm{H}_{2} \mathrm{O}$ transitions (e.g., Figure 4 of Pontoppidan et al. 2010).

To measure the strength of the $\mathrm{HCN}$ and $\mathrm{H}_{2} \mathrm{O}$ emission features, we used synthetic slab models of the molecular emission from disks that include $\mathrm{H}_{2} \mathrm{O}, \mathrm{OH}, \mathrm{CO}_{2}, \mathrm{HCN}$, and $\mathrm{C}_{2} \mathrm{H}_{2}$ (Carr \& Najita 2008, 2011) to identify continuum regions that are free of strong molecular emission. We identified continuum regions that bracket the $\mathrm{HCN}$ and $\mathrm{H}_{2} \mathrm{O}$ features of interest, as indicated in Figure 1. We then fit a linear continuum which we subtracted before measuring the flux of the water and $\mathrm{HCN}$ features. Because of the possibility that unresolved spectral features are present in either the feature or continuum regions, we interpret the measured values as 
emission strengths that are more akin to spectral indices than true fluxes.

At Spitzer resolution, the HCN feature may be blended with water emission (see Carr \& Najita 2011, Figure 4). To estimate the significance of the water contamination, we can use the fluxes of other bright water lines in the spectrum as a predictor of the strength of the water emission in the HCN region. We examined synthetic slab models of water emission (e.g., as described in Carr \& Najita 2011) as a guide to which water features best track the water emission strength in the HCN band. We adopted the estimator $0.4\left(F_{15.17}+0.8 F_{15.79}\right)$, where $F_{15.17}$ and $F_{15.79}$ are the emission strengths of the $15.17 \mu \mathrm{m}$ and $15.79 \mu \mathrm{m}$ water features, which predicts the contaminating water emission to within $\pm 20 \%$ over the range of temperatures (500-700 K) and column densities $\left(\log \left[N_{\mathrm{H}_{2} \mathrm{O}} / \mathrm{cm}^{-2}\right]=17.5-18.5\right)$ found for water emission from T Tauri disks (Carr \& Najita 2011; Salyk et al. 2011).

To estimate the strength of the HCN emission alone, we subtracted the estimated water emission in the $\mathrm{HCN}$ region using the above relation. The results discussed below are not sensitive to how this correction is made or whether it is carried out at all, as discussed in the following section. ${ }^{6}$ The predicted strength of the $\mathrm{H}_{2} \mathrm{O}$ emission in the $\mathrm{HCN}$ region is typically $\sim 20 \%$ of the measured $\mathrm{HCN}$ emission.

The emission strengths of HCN (before and after correction) and $\mathrm{H}_{2} \mathrm{O}$ are given in Table 1 . The tabulated uncertainties only account for the contribution from the noise in the spectrum, whereas the use of a linear fit to the underlying continuum contributes additional uncertainty. For example, the inability to account for dust emission features with our simple method can lead to negative emission strengths in some cases (e.g., FN Tau, HQ Tau).

Figure 2, which compares the $\mathrm{H}_{2} \mathrm{O}$ emission strength against the estimated $\mathrm{HCN}$ emission strength including the correction for contaminating water emission, shows that these are well correlated. The Kendall rank correlation test ( 28 sources) has a $\tau$ of 0.57 and 0.69 with and without the correction for contaminating water emission, respectively. The associated twosided $P$-values are $\sim 2 \times 10^{-5}$ or smaller. The higher correlation value for uncorrected $\mathrm{HCN}$ emission strength probably results from water emission in the $\mathrm{HCN}$ region which is correlated with the $17 \mu \mathrm{m}$ water emission that we measure. A related result, the correlation of $\mathrm{HCN}$ peak/continuum and $\mathrm{H}_{2} \mathrm{O} 17.2 \mu \mathrm{m}$ peak/continuum, was shown in Salyk et al. (2011).

We might expect a correlation between $\mathrm{HCN}$ and $\mathrm{H}_{2} \mathrm{O}$ emission from a disk atmosphere for several reasons. Increased heating (mechanical or radiative) would tend to produce a warmer disk atmosphere (i.e., with a higher temperature and possibly a larger emitting area) or a deeper temperature inversion in the atmosphere, which would increase the emission from all molecules that are present in it. The removal of grains from the atmosphere through settling can also increase the column density of the warm atmosphere that can be seen in emission. A lower abundance of grains renders a larger column density of the disk atmosphere optically thin in the continuum, thereby "revealing" its emission. In addition, if the heating of the disk

\footnotetext{
6 A similar, but not identical, method of measuring the $\mathrm{HCN}$ and $\mathrm{H}_{2} \mathrm{O}$ fluxes was used in Carr \& Najita (2011). The $\mathrm{H}_{2} \mathrm{O}$ fluxes reported there are the combined flux of same the three $\mathrm{H}_{2} \mathrm{O}$ features measured here. However, in the earlier study, the $\mathrm{H}_{2} \mathrm{O}$ flux was measured in the entire spectral swath between $17.075 \mu \mathrm{m}$ and $17.385 \mu \mathrm{m}$. The HCN flux was measured over the same spectral region, between $13.9 \mu \mathrm{m}$ and $14.05 \mu \mathrm{m}$, but after subtracting the best-fit slab emission models for $\mathrm{H}_{2} \mathrm{O}, \mathrm{OH}$, and $\mathrm{C}_{2} \mathrm{H}_{2}$, a more time-consuming approach. These details are unimportant to the results discussed here.
}

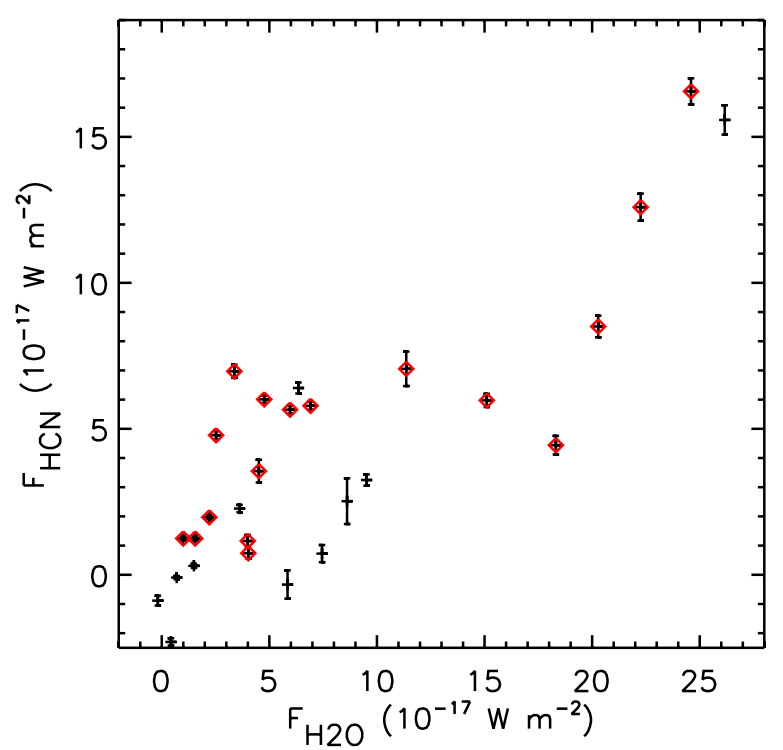

Figure 2. Emission strength of the $17 \mu \mathrm{m} \mathrm{H}_{2} \mathrm{O}$ emission features plotted against $\mathrm{HCN}$ emission strength. These quantities are well correlated, although there is significant dispersion, indicating the role of other parameters in determining the HCN emission strength. Red diamonds indicate sources with measured disk masses that are typical T Tauri stars, i.e., they have the mid-infrared colors of normal $\mathrm{T}$ Tauri stars and do not have a strong jet.

(A color version of this figure is available in the online journal.)

atmosphere has significant contributions from processes other than (grain-related) photoelectric heating, the removal of grains also reduces gas-grain cooling and warms the atmosphere. These effects would also increase the emission from all molecules that are present in the atmosphere.

Grain settling can, in principle, also alter the relative flux from molecular species that coexist in a disk atmosphere. As deeper layers of the disk atmosphere become visible as grains settle, not only is the visible column density of each species increased (as described above), but also, molecular level populations are thermalized at different depths as denser layers, deeper in the disk, are reached. Because species with higher critical densities are thermalized at greater depth, an apparent change in molecular flux ratios may arise as deeper regions of the disk are probed.

\section{2. $\mathrm{HCN} / \mathrm{H}_{2} \mathrm{O}$ Ratio and Disk Mass}

Although neither $\mathrm{HCN}$ (corrected for $\mathrm{H}_{2} \mathrm{O}$ contamination or not) nor $\mathrm{H}_{2} \mathrm{O}$ shows a trend individually with disk mass (Figure 4), the ratio of these quantities does show a trend with disk mass. Figure 3 plots the ratio of the (corrected) HCN to $\mathrm{H}_{2} \mathrm{O}$ emission strengths against disk mass. Sources with $\mathrm{H}_{2} \mathrm{O}$ emission strengths that are negative (HQ Tau) or near zero (FN Tau) were excluded from the plot. To highlight the properties of typical T Tauri stars, sources with a strong jet (DG Tau) or midinfrared colors outside the "Normal T Tauri" region of Furlan et al. $\left(2006 ; n_{13-25}>0.5\right)$ are not marked in red. The ratio of $\mathrm{HCN} / \mathrm{H}_{2} \mathrm{O}$ emission strength increases by at least an order of magnitude over a range of $\sim 100$ in disk mass.

The results confirm the possible trend between the $\mathrm{HCN} / \mathrm{H}_{2} \mathrm{O}$ emission ratio and disk mass shown in Carr \& Najita (2011). For the sources marked in red (17 sources), the Kendall rank $\tau$ is 0.61 with an associated two-sided $P$-value of $6 \times 10^{-4}$. A similar trend is found between the $\mathrm{HCN} / \mathrm{H}_{2} \mathrm{O}$ emission ratio and the $850 \mu \mathrm{m}$ flux; this is expected because the $850 \mu \mathrm{m}$ flux is a rough predictor of disk mass while a more precise measurement 


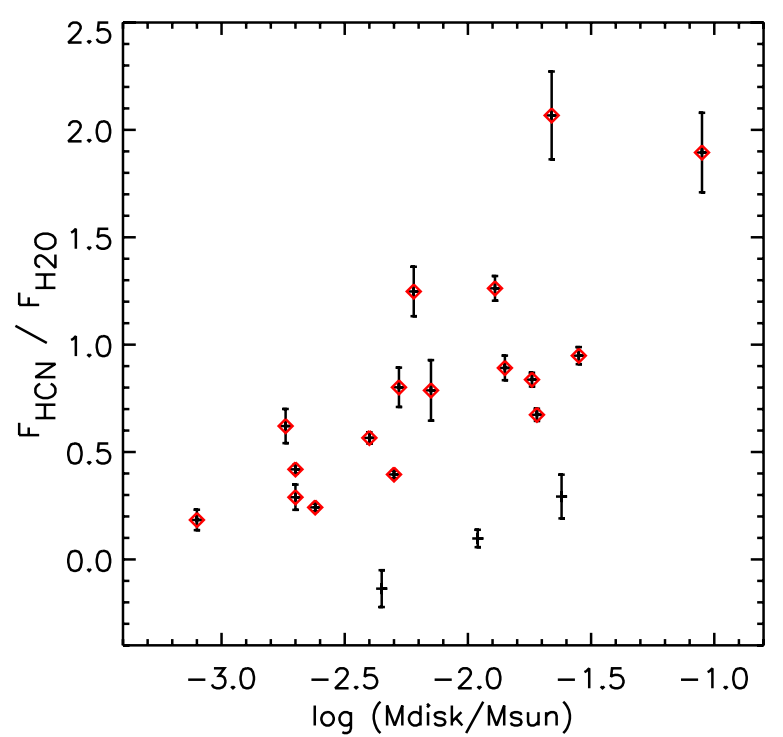

Figure 3. Ratio of the $\mathrm{HCN}$ and $\mathrm{H}_{2} \mathrm{O}$ emission strengths plotted against submillimeter disk mass. Red diamonds indicate sources with measured disk masses that are typical T Tauri stars, i.e., they have the mid-infrared colors of normal $\mathrm{T}$ Tauri stars and do not have a strong jet.

(A color version of this figure is available in the online journal.)

is obtained by modeling the SED over a wider range of wavelengths (Andrews \& Williams 2005). The correction to the HCN flux for contaminating water emission makes no significant difference to this result; using the uncorrected $\mathrm{HCN}$ strengths gives a Kendall rank $\tau$ of 0.58 and a two-sided $P$-value of $1 \times 10^{-3}$.

One might wonder if the trend with disk mass really reflects a trend with stellar mass, as $M_{*}$ may be proportional to $M_{\text {disk }}$ in an average sense. This is implausible because our sample spans a narrow range of spectral types (Table 1) and hence stellar masses ( $\sim 0.5-1.2 M_{\odot}$, e.g., White \& Ghez 2001). We also confirmed that the observed trend is not driven by mid-infrared color (cf. Section 3.1). Subsamples with redder or bluer mid-infrared color separately show the same trend between $\mathrm{HCN} / \mathrm{H}_{2} \mathrm{O}$ and disk mass seen in Figure 3.

Given the trend between disk mass and the $\mathrm{HCN}$ to $\mathrm{H}_{2} \mathrm{O}$ emission ratio, it is worth considering what these quantities measure. Within the context of homogeneous LTE slab models of these features (Salyk et al. 2011; Carr \& Najita 2011), the molecular fluxes depend on the column density, temperature, and emitting area for each molecule. For $\mathrm{H}_{2} \mathrm{O}$, the IRS spectrum contains both optically thick and optically thin lines over a range of excitation energies; as a result, we can characterize the typical temperature, column density, and area of the emitting region. The LTE slab modeling finds similar $\mathrm{H}_{2} \mathrm{O}$ column densities and temperatures for the sources studied. The range in $\mathrm{H}_{2} \mathrm{O}$ emission strength is therefore primarily a function of the $\mathrm{H}_{2} \mathrm{O}$ emitting area. In such a model, the total $\mathrm{H}_{2} \mathrm{O}$ mass contributing to the emission is the column density times the emitting area. Thus, to first order, the $\mathrm{H}_{2} \mathrm{O}$ flux provides a measure of the emitting mass of $\mathrm{H}_{2} \mathrm{O}$.

For HCN, there is some degeneracy between the column density and area of the emitting region. However, because the emission is optically thin (or only marginally optically thick), the HCN emission flux is roughly proportional to the HCN emitting mass. Therefore, the LTE slab modeling suggests that the $\mathrm{HCN} / \mathrm{H}_{2} \mathrm{O}$ flux ratio provides an approximate measure of the relative emitting masses of $\mathrm{HCN}$ and $\mathrm{H}_{2} \mathrm{O}$ in the inner disk.

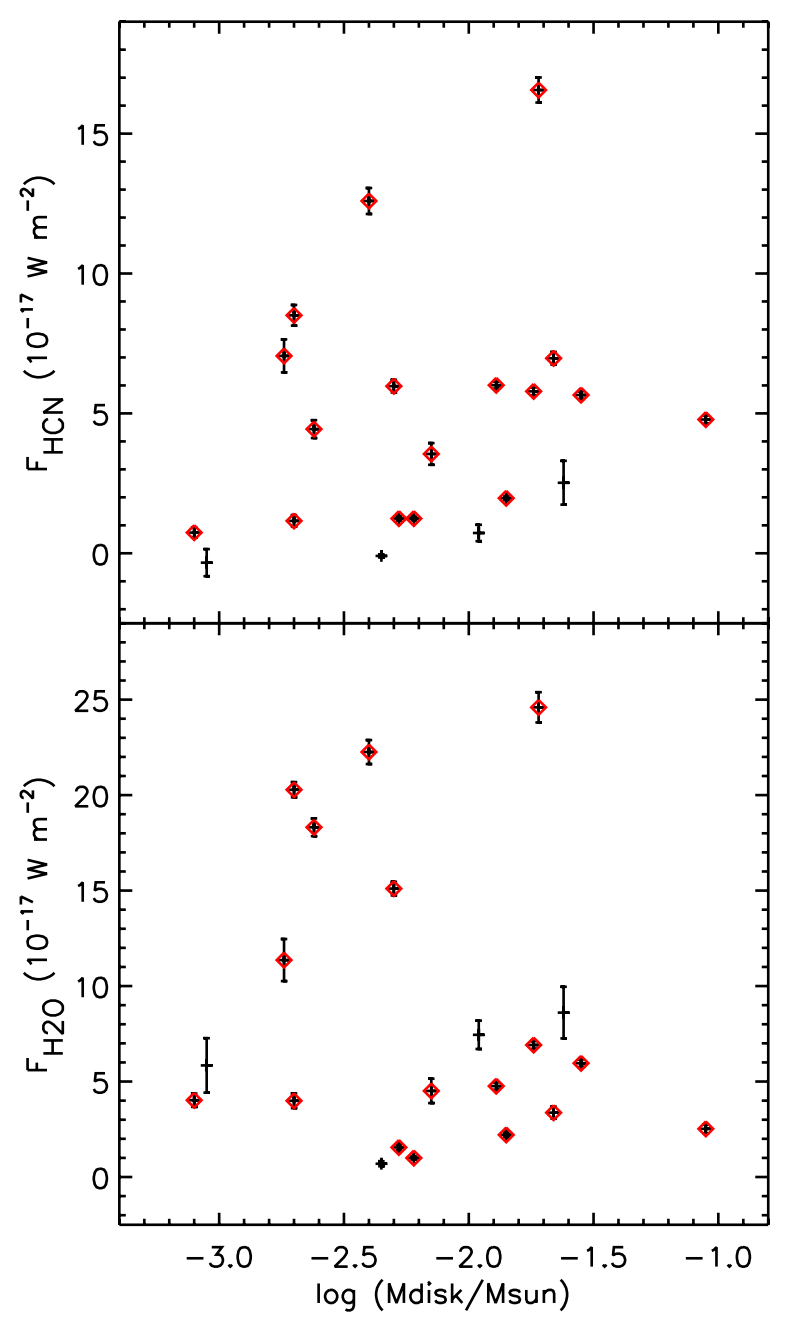

Figure 4. $\mathrm{HCN}$ (top) and $\mathrm{H}_{2} \mathrm{O}$ (bottom) emission strengths plotted against submillimeter disk mass. Red diamonds have the same meaning as in Figure 3. No trends are apparent, in contrast with Figure 3.

(A color version of this figure is available in the online journal.)

It is important to note, however, that sub-thermal collisional excitation of both $\mathrm{H}_{2} \mathrm{O}$ and $\mathrm{HCN}$ is probable (see Meijerink et al. 2009 for a discussion of non-LTE modeling of $\mathrm{H}_{2} \mathrm{O}$ ). If there are differences in the critical densities of the transitions that make up the $\mathrm{H}_{2} \mathrm{O}$ and $\mathrm{HCN}$ features, then object to object differences in the density of the molecular emitting region could induce variations in the $\mathrm{HCN} / \mathrm{H}_{2} \mathrm{O}$ flux ratio. If this is the case, then the $\mathrm{HCN} / \mathrm{H}_{2} \mathrm{O}$ flux ratio could be more sensitive to the excitation conditions than to the relative amount of $\mathrm{HCN}$ and $\mathrm{H}_{2} \mathrm{O}$. In Section 4.2, we discuss whether this effect could produce the observed trend in $\mathrm{HCN} / \mathrm{H}_{2} \mathrm{O}$ flux with disk mass.

We might similarly consider what disk mass measures. As noted above, the disk mass plotted in Figure 3 is based on submillimeter dust emission, which is sensitive to the mass of dust in the outer disk (beyond $\sim 20 \mathrm{AU}$ ). In the next section we discuss why a property of the disk at such large radii might be related to a molecular emission property of the inner disk (within a few AU).

\section{DISCUSSION}

We find that the possible trend of $\mathrm{HCN} / \mathrm{H}_{2} \mathrm{O}$ emission strength with disk mass shown in Carr \& Najita (2011) is confirmed in a larger sample. The ratio of $\mathrm{HCN} / \mathrm{H}_{2} \mathrm{O}$ emission strength increases by at least an order of magnitude over a range 
of $\sim 100$ in disk mass. It is perhaps surprising to find such a strong correlation between a molecular emission property of the inner disk with a property of the disk at much larger radii (the submillimeter dust mass). This is more remarkable given the large number of processes that could plausibly play a role in determining the molecular emission strengths of inner disks (e.g., thermal, chemical, and photo-processes, excitation). There are few similarly clear trends between inner disk molecular emission from T Tauri stars and other system parameters (Carr \& Najita 2011; Salyk et al. 2011). A similar kind of trend (e.g., of molecular ratios with disk mass) has not been reported in millimeter studies of the outer regions of $\mathrm{T}$ Tauri disks (e.g., Öberg et al. 2011b).

\subsection{A Consequence of Planetesimal or Protoplanet Formation?}

We previously noted (Carr \& Najita 2011; Najita et al. 2011) that an increasing trend of $\mathrm{HCN} / \mathrm{H}_{2} \mathrm{O}$ with disk mass might be expected to arise as a consequence of the formation of icy planetesimals and protoplanets. In the core accretion picture of planet formation, planets are built from collisions of planetesimals, solids larger than a kilometer in size, which themselves form from the aggregation of smaller solids. In the giant planet region of the disk (from the $\mathrm{H}_{2} \mathrm{O}$ snow line at a few $\mathrm{AU}$ to $\sim 20 \mathrm{AU}$ ), the condensation of water ice onto grains removes a significant amount of oxygen from the gas phase; hence, the $\mathrm{C} / \mathrm{O}$ ratio of the gas will be higher than the bulk composition of the disk, and the $\mathrm{C} / \mathrm{O}$ ratio of the solids lower (see also Öberg et al. 2011a and their Figure 1). As described by Ciesla \& Cuzzi (2006), as icy solids grow in this region of the disk (the outer disk), their migration, or lack thereof, can lead to gaseous inner disks that are either enhanced or depleted in water and oxygen. Resulting changes in the $\mathrm{C} / \mathrm{O}$ ratio could affect the abundance of hydrocarbons in the inner disk (Najita et al. 2011).

More specifically, when icy grains in the outer disk are small enough to couple well to the gas, water that is frozen on these grains accretes along with the gas into the inner planet formation region. When these grains pass the snow line and reach the inner disk, the water is returned to the gas phase as the ices evaporate, and the $\mathrm{C} / \mathrm{O}$ ratio of the inner disk gas is representative of the disk as a whole. However, when icy solids grow to larger (approximately meter) size, the strong headwind drag that they experience leads to rapid inward migration relative to the gas. When these bodies pass the snow line, their ices evaporate, hydrating the inner disk and, in depositing water and oxygen, reduce the $\mathrm{C} / \mathrm{O}$ ratio there. In contrast, when icy material in the outer disk reaches planetesimal or larger size, headwind drag becomes inconsequential, and these objects cease to migrate, sequestering water and oxygen beyond the snow line. As a result, the water- (and oxygen-) poor material that accretes into the inner disk enhances the $\mathrm{C} / \mathrm{O}$ ratio there.

These ideas suggest that early in the disk evolution process (before grain growth has occurred), the gaseous $\mathrm{C} / \mathrm{O}$ ratio of the inner disk will reflect the bulk $\mathrm{C} / \mathrm{O}$ ratio of the disk. At an intermediate stage, the inner disk will have a lower $\mathrm{C} / \mathrm{O}$ ratio than the disk as a whole. At a late stage, the inner disk will have a higher $\mathrm{C} / \mathrm{O}$ ratio than the disk as a whole.

In modeling the effect of the migration of solids on the evolution of the water distribution in protoplanetary disks, Ciesla \& Cuzzi (2006) found, in the specific scenarios they considered, that the gaseous water (and oxygen) abundance at
AU distances could be enhanced or depleted by a factor of 10 in either direction depending on the initial conditions and efficiency with which solids grow to large sizes. Such large variations would induce significant changes in the molecular abundances of the inner disk.

In their study of the thermal-chemical structure of inner disk atmospheres, Najita et al. (2011) looked at the effect of varying the inner disk $\mathrm{C} / \mathrm{O}$ ratio on the column density of warm molecules in the disk atmosphere, i.e., the column that could produce the molecular emission observed with Spitzer. They found that increasing the $\mathrm{C} / \mathrm{O}$ ratio of the inner disk from its nominal value of 0.4 to 0.67 was enough to raise the ratio of the warm column of $\mathrm{HCN}$ to that of $\mathrm{H}_{2} \mathrm{O}$ by an order of magnitude, similar to the range in the $\mathrm{HCN} / \mathrm{H}_{2} \mathrm{O}$ emission ratio seen in Figure 3 . The ratio increases dramatically because of both an increasing $\mathrm{HCN}$ abundance and a declining water abundance with increasing $\mathrm{C} / \mathrm{O}$. If the $\mathrm{HCN} / \mathrm{H}_{2} \mathrm{O}$ emission ratios that we measure are related to their molecular abundances, these disk chemistry results suggest that variations in the $\mathrm{C} / \mathrm{O}$ ratio of inner disks could plausibly explain the range of values we observe.

What role could disk mass play in such a scenario to produce the trend we see in Taurus? The relation in Figure 3 could be explained if more massive disks have locked up a greater fraction of their icy solids in non-migrating planetesimals and protoplanets, thereby increasing the $\mathrm{C} / \mathrm{O}$ ratio of their inner disks. This would imply that a significant fraction of the systems in Taurus are in the "late" stage of planetesimal formation described above. It seems reasonable that disks at the few Myr age of Taurus would be in this stage, if a significant fraction of these systems will go on to form giant planets within the few Myr gas dissipation timescale of disks.

More generally, in any given coeval star forming region, there may be a spread in how far systems have evolved along the "early-intermediate-late" sequence described above, with higher mass disks potentially evolving more quickly through this sequence. As a result, all star forming regions may not show the same trend seen here. In a younger star forming region, the reverse may occur: if the most massive disks are still in the "intermediate" stage and less massive disks are in the "early" stage, then higher mass disks would have inner disks with lower $\mathrm{C} / \mathrm{O}$ ratios.

In applying the above picture of planetesimal formation to the interpretation of Figure 3, we are assuming that disk masses estimated from submillimeter measurements (which probe disk radii beyond $20 \mathrm{AU}$ ) provide a useful indicator of the initial conditions in the giant planet formation region (from the snow line to $20 \mathrm{AU}$ ), i.e., the region where icy planetesimals could form in sufficient abundance to affect the $\mathrm{C} / \mathrm{O}$ ratio of the inner disk. Because grain evolution slows greatly with increasing disk radius (e.g., Dullemond \& Dominik 2005; Pérez et al. 2012), it seems plausible that the current disk region beyond $\sim 20 \mathrm{AU}$ would provide some insight into the mass that was originally present in the few-20 AU region, at least in a relative sense. Our interpretation that the trend of $\mathrm{HCN} / \mathrm{H}_{2} \mathrm{O}$ with submillimeter disk mass reflects the enhanced formation of large icy bodies in more massive disks only assumes that the submillimeter disk mass gives a relative ordering of the initial mass in the planet formation region of the disk. This perspective is similar to that adopted in other studies that assume (and argue why) submillimeter fluxes can be used to infer the planet-forming potential of a given disk (e.g., Greaves et al. 2007; Wyatt et al. 2007).

This suggested interpretation highlights the possibility of looking for chemical signatures of observationally elusive steps 
in the planet formation process. A related idea has been explored in Öberg et al. (2011a) regarding the effect of snow lines on the $\mathrm{C} / \mathrm{O}$ ratio in planetary atmospheres. They investigated how the different $\mathrm{C} / \mathrm{O}$ ratios of the disk gas and solids as a function of disk radius can lead to different $\mathrm{C} / \mathrm{O}$ ratios in the atmospheres of giant planets depending on how and where the planets acquired their atmospheres. As a result, the $\mathrm{C} / \mathrm{O}$ ratio of the atmospheres of giant planets may provide a valuable chemical clue to that step in the planet formation process.

\subsection{Alternative Explanation: Subthermal Excitation of HCN?}

In Section 3 we described how the results of earlier LTE slab modeling suggest that the $\mathrm{HCN} / \mathrm{H}_{2} \mathrm{O}$ flux ratio can probe the relative masses of $\mathrm{HCN}$ and $\mathrm{H}_{2} \mathrm{O}$ in the molecular emission region. It is important to examine the assumption of LTE, because non-LTE conditions might provide an alternative explanation for the trend we see. If $\mathrm{HCN}$ were subthermally excited relative to $\mathrm{H}_{2} \mathrm{O}$, and if higher disk masses mean higher disk densities overall, then the $\mathrm{HCN} / \mathrm{H}_{2} \mathrm{O}$ emission ratio might increase with disk mass simply because $\mathrm{HCN}$ is better excited, not because it is more abundant.

The $\mathrm{HCN}-\mathrm{H}_{2}$ collisional rate coefficients at $300 \mathrm{~K}$ (and higher) for the $\nu_{2}$ bending mode are $\sim 10^{-11} \mathrm{~cm}^{3} \mathrm{~s}^{-1}$ (Smith $\&$ Warr 1991). For a typical Einstein $A$-value of $3 \mathrm{~s}^{-1}$, the HCN critical density is $\sim 2 \times 10^{11} \mathrm{~cm}^{-3}$. In comparison, collisional rate coefficients for $\mathrm{H}_{2} \mathrm{O}-\mathrm{H}_{2}$ correspond to critical densities of $10^{9}-10^{11} \mathrm{~cm}^{-3}$, depending on the transition. The densities in the warm atmosphere at the radii from which the molecular emission is believed to arise (0.25-1 AU) are estimated to be $\sim 10^{10}-10^{12} \mathrm{~cm}^{-3}$ and vary in both vertical height and radius (e.g., in the models of Najita et al. 2011). Hence, HCN could be subthermally excited relative to $\mathrm{H}_{2} \mathrm{O}$ over some portion of the molecular emitting region. Detailed calculations are needed to understand whether and how strongly the $\mathrm{HCN} / \mathrm{H}_{2} \mathrm{O}$ emission ratio is affected by the density of the disk atmosphere.

While density could play a role in the measured $\mathrm{HCN} / \mathrm{H}_{2} \mathrm{O}$ emission flux ratio, it is unclear whether this could produce a correlation with disk mass. In order to produce the relation in Figure 3, the submillimeter disk mass (which probes the region beyond $20 \mathrm{AU}$ ) would have to be a good indicator of the density of the inner disk atmosphere (within a few AU) where the molecular emission arises. The possibility of non-steady disk accretion, dead zones, and accretion outbursts suggests that the masses that currently reside in the inner and outer disks might not be well correlated. ${ }^{7}$ Moreover, even if the inner disk mass is correlated with the outer disk mass, the density in the molecular line formation region of the upper atmosphere may not be as coupled. As noted in Section 3.2, the temperature and column density of the warm disk atmosphere, as probed by the water emission, appears to be similar among T Tauri disks (Carr \& Najita 2011; Salyk et al. 2011). This suggests that the density in the warm atmosphere does not vary greatly. We discuss this topic and other possibilities for future work in the following section.

\section{CONCLUSIONS AND FUTURE WORK}

We find a clear relation between the mid-infrared $\mathrm{HCN} / \mathrm{H}_{2} \mathrm{O}$ flux ratio and submillimeter disk mass among $\mathrm{T}$ Tauri stars in Taurus. It is perhaps surprising that emission properties of the

\footnotetext{
7 This skepticism can be distinguished from our earlier assumption that the outer disk (beyond $20 \mathrm{AU}$ ) might reasonably probe the initial rather than current mass of the giant planet region (few-20 AU).
}

gas in the inner disk ( $<$ a few $\mathrm{AU}$ ) are related to a property (disk mass) probed by the dust in the outer disk (beyond $20 \mathrm{AU}$ ).

An interesting possible interpretation is that the trend arises as a consequence of the formation of icy planetesimals and protoplanets. In this scenario, higher mass disks more readily form such large (non-migrating) bodies, which lock up water ice (and oxygen) beyond the snow line. The water- (and oxygen-) poor material that reaches the inner disk enhances the $\mathrm{C} / \mathrm{O}$ ratio of the gas there. Because a higher $\mathrm{C} / \mathrm{O}$ ratio both enhances the abundance of $\mathrm{HCN}$ and reduces the abundance of $\mathrm{H}_{2} \mathrm{O}$, the $\mathrm{HCN} / \mathrm{H}_{2} \mathrm{O}$ ratio can rise dramatically as a result.

This interpretation requires that (1) the $\mathrm{HCN} / \mathrm{H}_{2} \mathrm{O}$ flux ratio is sensitive to the relative masses of $\mathrm{HCN}$ and $\mathrm{H}_{2} \mathrm{O}$ in the molecular emission region; (2) submillimeter disk masses are a reasonable indicator of the initial masses in the icy planetesimal and protoplanet formation region of the disk (few to $~ 20 \mathrm{AU}$ ); and (3) higher mass disks in Taurus have formed non-migrating icy planetesimals more efficiently or more rapidly than lower mass disks.

An alternative explanation is that the variations in the $\mathrm{HCN} / \mathrm{H}_{2} \mathrm{O}$ flux ratio are instead an excitation effect related to density. This would require that the density of the warm molecular layer of the inner disk where the emission arises is correlated with the mass of the outer disk as measured by submillimeter dust emission.

We can explore these ideas in several ways. On the observational and modeling end, we need to measure relative molecular masses (rather than fluxes). Currently, the errors on the molecular column density (or mass) estimates from LTE analysis for $\mathrm{HCN}$ and $\mathrm{H}_{2} \mathrm{O}$ (Carr \& Najita 2011; Salyk et al. 2011) are too large to measure relative molecular masses. The higher spectral resolution $(R=3000)$ of MIRI on the James Webb Space Telescope or infrared echelle spectroscopy on future large telescopes can potentially resolve the modeling degeneracies that arise from the low-resolution Spitzer spectra.

These measurements should be combined with a detailed nonLTE model for the excitation of $\mathrm{HCN}$ and $\mathrm{H}_{2} \mathrm{O}$. With such a tool, one could also explore the counterhypothesis, that the observed trend is a result of subthermal excitation of HCN compared to $\mathrm{H}_{2} \mathrm{O}$, by looking for evidence of increased subthermal excitation of $\mathrm{HCN}$ and $\mathrm{H}_{2} \mathrm{O}$ in sources with lower disk masses. We should also search for trends involving other molecular ratios that would be affected by variations in the $\mathrm{C} / \mathrm{O}$ ratio of the inner disk (possibly $\mathrm{C}_{2} \mathrm{H}_{2} / \mathrm{H}_{2} \mathrm{O}$; e.g., Najita et al. 2011). On the theory end, we need a more complete model of the formation of planetesimals and protoplanets, their efficiency of formation as a function of disk mass, and their effect on the transport of water in the disk, in order to properly evaluate whether our proposed interpretation of the trend of $\mathrm{HCN} / \mathrm{H}_{2} \mathrm{O}$ with disk mass is tenable.

This work is based on observations made with the Spitzer Space Telescope, which is operated by the Jet Propulsion Laboratory, California Institute of Technology under a contract with NASA. Support for this work was provided by NASA. Basic research in infrared astrophysics at the Naval Research Laboratory is supported by 6.1 base funding. J.N. thanks Sharmila Dey for assistance with the data analysis.

\section{REFERENCES}

Agúndez, M., Cernicharo, J., \& Goicoechea, J. R. 2008, A\&A, 483, 831 Andrews, S. M., \& Williams, J. P. 2005, ApJ, 631, 1134 Carr, J. S., \& Najita, J. R. 2011, ApJ, 733, 102 
Carr, J. S., \& Najita, J. R. 2008, Sci, 319, 1504

Chiang, E., \& Youdin, A. 2010, AREPS, 38, 493

Ciesla, F. J., \& Cuzzi, J. N. 2006, Icar, 181, 178

Dullemond, K., \& Dominik, C. 2005, A\&A, 434, 971

Evans, N. J., II, Allen, L. E., Blake, G. A., et al. 2003, PASP, 115, 965

Furlan, E., Hartmann, L., Calvet, N., et al. 2006, ApJS, 165, 568

Greaves, J. S., Fischer, D. A., Wyatt, M. C., Beichman, C. A., \& Bryden, G. 2007, MNRAS, 378, L1

Hartigan, P., Strom, K. M., \& Strom, S. E. 1994, ApJ, 427, 961

Heinzeller, D., Nomura, H., Walsh, C., \& Millar, T. J. 2011, ApJ, 731, 115

Hillenbrand, L. A. 2008, PhyS, 130, 014024

Houck, J. R., Roellig, T. L., van Cleve, J., et al. 2004, ApJS, 154, 18

Itoh, Y., Tamura, M., Hayashi, M., et al. 2008, PASJ, 60, 209

McCabe, C., Duchêne, G., \& Ghez, A. M. 2003, ApJL, 588, L113

Meijerink, R., Pontoppidan, K. M., Blake, G. A., Poelman, D. R., \& Dullemond, C. P. 2009, ApJ, 704,1471
Najita, J. R., Ádámkovics, M., \& Glassgold, A. E. 2011, ApJ, 743, 147

Öberg, K. I., Murray-Clay, R., \& Bergin, E. A. 2011a, ApJL, 743, L16

Öberg, K. I., Qi, C., Fogel, J. K. J., et al. 2011b, ApJ, 734, 98

Pascucci, I., Apai, D., Luhman, K., et al. 2009, ApJ, 696, 143

Pérez, L. M., Carpenter, J. M., Chandler, C. J., et al. 2012, ApJL, 760, L17

Pontoppidan, K. M., Salyk, C., Blake, G. A., et al. 2010, ApJ, 720, 887

Salyk, C., Pontoppidan, K. M., Blake, G. A., Najita, J. R., \& Carr, J. S. 2011, ApJ, 731,130

Salyk, C., Pontoppidan, K. M., Blake, G. A., et al. 2008, ApJL, 676, L49

Skemer, A. J., Close, L. M., Greene, T. P., et al. 2011, ApJ, 740, 43

Smith, I., \& Warr, J. 1991, J. Chem. Soc. Far. Trans., 87, 807

White, R. J., \& Ghez, A. M. 2001, ApJ, 556, 265

Willacy, K., \& Woods, M. 2009, ApJ, 703, 479

Woitke, P., Kamp, I., \& Thi, W.-F. 2009, A\&A, 501, 383

Wyatt, M. C., Clarke, C. J., \& Greaves, J. S. 2007, MNRAS, 380, 1737 